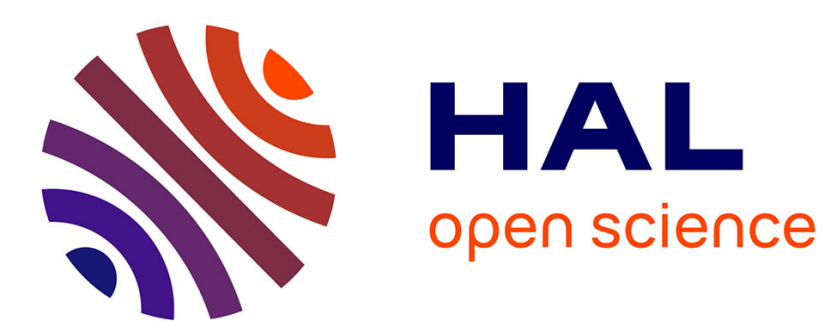

\title{
Dislocation motion : a wavenumber selection mechanism in Rayleigh-Benard convection
}

\author{
A. Pocheau, V. Croquette
}

\section{To cite this version:}

A. Pocheau, V. Croquette. Dislocation motion: a wavenumber selection mechanism in RayleighBenard convection. Journal de Physique, 1984, 45 (1), pp.35-48. 10.1051/jphys:0198400450103500 . jpa-00209738

\section{HAL Id: jpa-00209738 https://hal.science/jpa-00209738}

Submitted on 1 Jan 1984

HAL is a multi-disciplinary open access archive for the deposit and dissemination of scientific research documents, whether they are published or not. The documents may come from teaching and research institutions in France or abroad, or from public or private research centers.
L'archive ouverte pluridisciplinaire HAL, est destinée au dépôt et à la diffusion de documents scientifiques de niveau recherche, publiés ou non, émanant des établissements d'enseignement et de recherche français ou étrangers, des laboratoires publics ou privés. 


\title{
Dislocation motion : a wavenumber selection mechanism in Rayleigh-Bénard convection
}

\author{
A. Pocheau and V. Croquette $\left({ }^{*}\right)$ \\ SPSRM, CEN Saclay, 91191 Gif sur Yvette, France \\ (Reçu le 14 janvier 1983, révisé le 29 août, accepté le 12 septembre 1983)
}

\begin{abstract}
Résumé. - Dans cet article, nous avons mesuré la vitesse de montée d'une dislocation isolée dans une structure de rouleaux de Rayleigh-Bénard de grande extension, en fonction du nombre d'onde et du nombre de Rayleigh. Le mouvement de la dislocation dans cette structure par ailleurs parfaite, constitue un mécanisme de sélection du nombre d'onde. D'autre part nous avons comparé ce mécanisme à celui obtenu à partir de défauts du type joints de grain. Ces deux mécanismes conduisent à une sélection univoque du nombre d'onde, ce qui a permis une détermination précise de la courbe de sélection.
\end{abstract}

\begin{abstract}
In this article, we report the measurement and analysis of the climbing velocity of an isolated dislocation in a Rayleigh-Bénard roll structure of large extension, as a function of the wavenumber and of the Rayleigh number. The motion of this dislocation in the otherwise regular structure is found to be a wavenumber selection mechanism. Further, we compare this mechanism with that provided by grain boundary defects. Both mechanisms lead to a wavenumber selection consisting of a unique wavevector rather than a band, which allows the selection curve to be accurately determined.
\end{abstract}

\section{Introduction.}

The Rayleigh-Bénard instability provides at least two different experimental situations for studying the occurrence of turbulence : the first deals especially with its temporal feature and appears in so called small aspect-ratio containers ; the second, which occurs in the large aspect-ratio case also involves the temporal aspect but it is closely connected to the spatial character of turbulence. The aspect-ratio $G$ of the container is the ratio of its length to its depth.

When $G$ is small the structure contains few rolls. Their positions are then strictly defined and the turbulence which occurs is closely related to the established convective pattern [1]. It is not surprising then that this turbulence can be described by model systems involving a small number of degrees of free$\operatorname{dom}[2]$.

However, in large aspect-ratio containers, the structure is by no means so constrained and the convective patterns appear to be disordered even in the simple roll convective state [3-5]. When the convection has just set in, two behaviours may be encountered : the first one is characterized by a slow relaxation of the

(*) Mail should be sent to this author. structure towards a stationnary state which is also disordered [3, 4], so that turbulence only occurs at a high Rayleigh number, generally through the appearence of another instability. The second behaviour is characterized by the fact that these structures present a permanent time evolution $[6,7]$, that is, the flow is turbulent as soon as the convection appears. The difference between these behaviours is related to the properties of the fluid under study, and more precisely to its Prandtl number, $P=v / \kappa$.

Nevertheless, very little experimental data about this kind of turbulence is presently available. In the experiment of reference 6 , only the equivalent of the global heat flux was recorded, while in [7] a local temperature was analysed. Furthermore, in both experiments, the convective pattern remained unknown since no visualization could be achieved. However the bare possibility of this turbulence has motivated theoreticians to reconsider the supercritical convective state consisting of simple rolls. In a previous approach, the convective roll pattern was considered to be analogous to a crystal which is periodic in only one direction. Such a "crystal " can be modelled by an amplitude equation which describes the dynamics of its modes of perturbation [8-10]. As for ordinary three dimensional crystals, these equations 
are derived from a variational principle and can only account for a relaxational behaviour $[11,12]$. In a later and more detailed approach, the non-variational terms, whose contributions to the amplitude equations cannot be written as the gradient of any potential (just as the Boussinesq equations themselves), were considered. These terms may perhaps account for the turbulent behaviour observed in references 6 and 7 .

Due to their relative simplicity when compared to the Boussinesq equations, amplitude equations have allowed a lot of predictions to be made concerning the essential properties of the convective roll patterns. For example, the old problem of the wavenumber selection has led to several specific predictions [13-17]. In addition, structural defects have been studied in detail : dislocation dynamics have been investigated in references 18 to 20 and grain boundaries in reference 21 .

A number of these predictions need comparison with experimental results, first in order to test the validity of the amplitude equations $[9,22]$ and second, to clarify the occurring mechanisms.

In this article, we present the first experimental study of the dynamics of isolated dislocations in Rayleigh-Bénard convection. In particular, we have measured their climbing velocity as a function of the Rayleigh number and of the wavevector. We demonstrate that dislocation climb provides an efficient wavevector selection mechanism and we have accurately determined the selected states. Indeed it is difficult to change the wavenumber in a perfect structure bounded by periodic conditions. Such a modification involves the occurrence of a structural instability or rather, more frequently, of the motion of a defect, e.g. a dislocation. In the same spirit, we have studied the wavenumber selection obtained with grain boundary defects and we have compared these two mechanisms. Finally, using our results, we have analysed the experimental results of $\mathbf{J}$. A. Whitehead.

\section{Essential features of defects in Rayleigh-Bénard convection.}

As an introduction to our experimental results, presented in section 3 , in this section we outline the main lines of defect studies in the Rayleigh-Bénard field.

It is now recognized that, in contradiction to the simple idea that convection should appear as perfectly regular patterns (parallel rolls in rectangular containers or axisymmetric patterns in cylindrical containers), " textured " structures are the general rule in large aspect ratio containers $[22,3,4]$. These structures consist of patches of fairly regular rolls with various orientations and dimensions linked together by structural defects such as dislocations, disclinations, or grain boundaries. These patterns have peculiar dynamics : they present long relaxation periods, during which the defects move, which leads to a reorganization of the entire structure, towards a stationary state [12].
In some cases, no stationary states are ever encountered so that these structures present a turbulent behaviour $[6,7]$. Since the relaxation towards a stationary state may easily be described as an evolution in a potential valley [11], as in any variational system, the turbulent behaviour which appears in references $[6,7]$ cannot be modelled in such a simple scheme and a non variational approach is required.

Experiments show that the dynamics of these structures are closely connected to those of defects. However it is not clear whether these disordered dynamics may be explained in terms of motion of isolated defects or, whether they have something to do with their interactions. Thus it seems interesting to consider first the dynamics of an isolated defect, and a good candidate for such a study is a dislocation.

This approach was first developed by E. D. Siggia and A. Zippelius in a theoretical study [18]. Their original motivation was to explain defect motions having noticed the peculiar rôle played by the vertical vorticity. This idea was suggested by J. A. Whitehead's experiment [23] which consisted in the study of the motion of a row of dislocations in a parallel roll structure. The velocity of this motion was found to scale like $P^{-1}$ just as the vertical vorticity which always appears together with dislocations. As the amplitude equation [8-10] neglects the vertical vorticity, the dislocation velocity was not expected to be properly described by it. However they showed that this amplitude equation was sufficient to describe resonably well the motion of dislocations in the limit of a high Prandtl number. But they indicated that the case of a low Prandtl number was not yet understood and that the vertical vorticity could be responsible for a turbulent convective behaviour. In this spirit they proposed a new amplitude equation which explicitly takes into account this variable [24]. This would be a way to incorporate non-variational aspects of convection into the amplitude equation and thus to investigate their effect on dislocation motion.

In their article [18], Siggia and Zippelius have considered the case of dislocations placed in the middle of a parallel roll structure. Due to the symmetry of this structure, only climbing motion can occur. They have investigated this motion using three approaches : first by direct simulations of the Boussinesq equations, second by simulations of the amplitude equation, and third by an analytic calculation based on the amplitude equation of reference 10 . The main result of this last calculation is an expression of the climbing velocity :

$$
V=\beta \frac{\xi_{\|}^{2}}{\tau} \frac{k_{\mathrm{c}}}{\sqrt{2}}\left(\frac{\mathrm{d} k}{k_{\mathrm{c}}}\right)^{3 / 2}
$$

with

$$
\xi_{\|}^{2}=0.148 d^{2} / \varepsilon
$$


and

$$
\tau=\frac{d^{2}}{\kappa} \cdot \frac{0.5117+P}{19.65 \varepsilon P}, \quad \mathrm{~d} k=k-k_{\mathrm{c}}
$$

so that :

$$
V=\beta \frac{\kappa}{d} \cdot \frac{6.4098}{1+0.5117 P^{-1}}\left(\frac{\mathrm{d} k}{k_{\mathrm{c}}}\right)^{3 / 2}
$$

where $\mathrm{d} k$ is the distance between the average wavevector $k$ of the structure and the critical one $k_{\mathrm{c}}$, $\beta$ a numerical constant, $\tau$ the characteristic time of the convective layer, $\xi_{\|}$the influence length and $d$ the depth of the fluid layer. This velocity corresponds to the expansion of the structure having the wavenumber nearest to the critical one.

Thus dislocation motion appears to be a wavenumber selection mechanism with the critical wavevector $k_{\mathrm{c}}$ as the optimal one. We may notice that in the above expression the velocity does not depend on the reduced Rayleigh number $\varepsilon=\left(R-R_{\mathrm{c}}\right) / R_{\mathrm{c}}$. This result significantly differs from the findings of J. A. Whitehead, who showed that a row of dislocations has a velocity roughly proportional to $\varepsilon$. As will be discussed in part 3, Whitehead's experiment does not correspond to the domain of validity of the calculations of Siggia and Zippelius. We think that this discrepancy is inherent to the framework of their calculations, which rely on the amplitude and phase equations proposed by Newell and Whitehead [10], who derived them at the lowest order in $\varepsilon$ for stress-free boundary conditions, that is $\sqrt{\varepsilon}$ for the velocity field.

At this order Zippelius and Siggia have recently demonstrated [24] that large scale flows, which appear at order $\varepsilon$, significantly modify the amplitude equation of reference 10 . The major consequence of using the Newell-Whitehead equation is that it describes the phenomenon of the wavenumber selection only to order $\sqrt{\varepsilon}$. That is to say, the preferred wavevector is the critical one $k_{\mathrm{c}}$ and the wavenumber selection is then independent of the Prandtl number $P$ or of the reduced Rayleigh number $\varepsilon$. Obviously this does not correspond to the experimental situation even in the limit of infinite Prandtl number. Our experiment proves (see section 3) that the velocity of the dislocations is clearly related to the wavenumber selection and that this is responsible for its dependence on $\varepsilon$.

In fact Siggia and Zippelius could have been aware of this problem since, as shown by their results concerning the Boussinesq simulation, which indeed represents the most realistic case, the velocity of the dislocation increases significantly with $\varepsilon$ at a defined wavevector.

On the other hand, their simulation of the amplitude equation, which ought to reproduce their analytical calculations based on the same equations, shows that the dislocation velocity decreases slightly with $\varepsilon$. This dependence of $V$ on $\varepsilon$, though small, is surprising since it is opposite to the evolution displayed by Boussinesq equation simulation. In both simulations, the dependence of $V$ on $\varepsilon$, although obvious when considering table III of [18], was not discussed.

As the amplitude equation contains the Prandtl number dependence only through scaling factors, the dislocation velocity calculated by E. D. Siggia and A. Zippelius depends on the Prandtl number in the same way as the scaling factors. Replacing once more Newell-Whitehead equations by more realistic ones should alter these results and describe the experimental situation more accurately.

The problem of dislocation motion has been reconsidered by Y. Pomeau, S. Zaleski and P. Manneville [19] in a slightly more general context which still does not take the vertical vorticity into account. They considered climbing as well as gliding motions in the two complementary cases, namely non-variational and variational, using model equations. In the latter case, they used an analogy with a crystal, so that the dislocation velocity simply appears as the balance between the viscous damping force and the Peach-Kohler force [25]. This force, equivalent to the elastic stresses acting on the dislocation, may be expressed in terms of the perpendicular diffusion coefficient $D_{\perp}$, and the dislocation velocity is found to behave as $\left(D_{\perp}\right)^{3 / 2}$

$$
V=\beta \frac{\tau^{1 / 2}}{\xi_{\|}} \frac{k_{\mathrm{c}}}{\sqrt{2}} D_{\perp}^{3 / 2}
$$

With

$$
D_{\perp}=\frac{\xi_{\|}^{2}}{\tau}\left[\frac{\mathrm{d} k}{k_{\mathrm{c}}}+N(P) \cdot \varepsilon\right] \text {. }
$$

If we conjecture that this expression remains meaningful even in the presence of a weak vertical vorticity, we may replace $N(P)$ by its expression [26] :

$$
N(P)=\frac{0.16591+(23.0935 / P)+\left(6.1961 / P^{2}\right)}{10.7579-(0.07256 / P)+\left(0.12805 / P^{2}\right)} \text {. }
$$

Such an assumption cannot be justified but seems interesting in the case of a relatively large Prandtl number.

This leads to the following expression for the dislocation velocity

$$
V=\beta \frac{\kappa}{d} \frac{6.4098}{1+0.5117 P^{-1}}\left[\frac{\mathrm{d} k}{k_{\mathrm{c}}}+N(P) \varepsilon\right]^{3 / 2}
$$

In these expressions, we have used the coefficient $\beta$ introduced by Siggia and Zippelius in [18], related to the $\beta^{\prime}$ used in [19] by : $\beta=2 \sqrt{2} \beta^{\prime}$.

As in [18], dislocation motion appears as a wavenumber selection mechanism since during their motion dislocations favour structures with the smaller $D_{\perp}$. 
The stationary states correspond then to the condition $D_{\perp}=0$, i.e. the condition of marginal stability against zigzags. Although mathematically different from the method of Siggia and Zippelius, this technique [19] uses the same kind of amplitude and phase diffusion equations. However, these equations have been developed up to second order in the reduced Rayleigh number, leading to a non-trivial dependence of the velocity on the Rayleigh number, contrary to what was found by E. D. Siggia and A. Zippelius. The diffusion coefficient $D_{\perp}$ has been derived up to this order as a linear function of the mean wavevector distance from the critical wavevector $\mathrm{d} k$ and of the reduced Rayleigh number $\varepsilon$ by $P$. Manneville and J. M. Piquemal [26]. Thus the selection curve of zero velocity is a line $\varepsilon=-6.94 \times \mathrm{d} k$ at $P=70$ and the selected wavenumber decreases with the Rayleigh number.

In model amplitude equations and in the no slip Rayleigh-Bénard case, the non-variational character appears at second order in the reduced Rayleigh number ; this is the order considered in this theoretical study [19]. The same mathematical derivation as in the variational case then leads to a similar velocity law and to an evaluation of non-variational effects. The main difference with the variational case is that the curve of zero velocity no longer coincides with $D_{\perp}=0$. However, as one moves further from the selected region, that is when $V$ becomes significant, the non-variational law becomes asymptotically close to the variational law so that $V$ scales roughly like $D_{\perp}^{3 / 2}$ and at some distance from the curve $V=0$, it is difficult to distinguish between the two behaviours.

From this point of view, the only observable non-variational effects take place close to the selection curves. However, the possible dependence on the Prandtl number due to vertical vorticity still remains inaccessible to these model equation treatments since as in [18], these equations do not take into account its occurrence at low Prandtl number. Furthermore, Pomeau et al. do not consider the Rayleigh-Bénard case explicitly but rather the simpler non-variational case of Darcy-Rayleigh thermoconvection.

The only reported experiment on dislocation dynamics in Rayleigh-Bénard structures was carried out by J. A. Whitehead [23]. As already mentioned, this experiment deals with a row of dislocations rather than with an isolated dislocation, so that it may not be directly compared with the theoretical studies [18, 19]. However, a remarkable experimental result was pointed out : the velocity of the row of dislocations was found constant in time. This point has been taken as an assumption in both theories $[18,19]$.

We have carried out an experiment involving the pattern evolution of a single and isolated dislocation in an otherwise ideal pattern. The general purpose of this experiment was to study the dislocation velocity as a function of the Rayleigh number and of the wavevector. This study has been greatly facilitated by two previous experiments : in reference 22 we investigated the dynamical properties of ideal structures $\left(D_{\|}, D_{\perp}\right)$ and in reference 3 we considered the properties of stationnary defects. However the present study points out the wavenumber selection problem since, as we shall see below, dislocation motion provides a mechanism of wavenumber selection. Another one consists in a continuous wavelength adjustement occurring with grain boundary defects. In this article we present the results of these two experiments, which we have carried out using the same container and the same fluid.

\section{Experimental configuration.}

The description of the experimental apparatus has already been presented elsewhere [22], and we only recall briefly here its main characteristics. The container used consists of a plexiglass frame sandwiched between a massive copper plate at the bottom and a sapphire monocrystal plate at the top. The temperature of both plates is controlled by water circulation, the accuracy obtained on the temperature difference being on the order of $0.02{ }^{\circ} \mathrm{C}$. In order to work with a large aspect-ratio container we have reduced its depth to $2.56 \mathrm{~mm}$, the horizontal dimensions being $98.25 \mathrm{~mm}$ and $60 \mathrm{~mm}$. Therefore we expect 19 wavelengths to be present in the cell for the critical wavevector. It is obvious that a small variation of this depth would produce a drastic modification of the wavevector as well as of the value of the Rayleigh number. In order to minimize this effect and to ensure a uniform depth over all the cell we have used three spacers placed between the copper plate and the sapphire. The plexiglass frame was glued on both the copper and sapphire surfaces, and the thickness of the glue layer was about $0.02 \mathrm{~mm}$. We estimate our depth homogeneity to be of the same order of magnitude.

We have performed our experiments with silicone oil having the following physical properties : a kinetic viscosity $v=5 \mathrm{cSt}\left(25^{\circ} \mathrm{C}\right)$, a thermal diffusivity $\kappa=7 \times 10^{-4} \mathrm{~cm}^{2} / \mathrm{s}\left({ }^{1}\right)$, and thus a Prandtl number of 70. This Prandtl number is interesting since it is sufficiently large to prevent oscillatory or varicose instabilities but is low enough so that the RayleighBénard convective parameters, such as the slope of the line $D_{\perp}=0$, still vary in a noticeable manner. With this experimental configuration, the convection threshold $R_{\mathrm{c}}$ was reached when the temperature difference was $4.85^{\circ} \mathrm{C}$. We have investigated convective structures from $R=R_{\mathrm{c}}$ to $R=5 R_{\mathrm{c}}$.

( $\left.{ }^{1}\right)$ The value of the thermal diffusivity of the $5 \mathrm{cSt}$ silicon oil is not available from the manufacturer. The value given here is an extrapolation from the thermal diffusivity of oils having a viscosity close to $5 \mathrm{cSt}$. Thus this value may be in error by 10 to $15 \%$, resulting in the same uncertainty on the Prandtl number. 
2. 1 DisLOCATION EXPERIMENT. - If we consider the structures which appear when we smoothly.increase the temperature difference above the convection threshold, that is if we consider " natural structures » $[22,3]$ we observe patterns very similar to that shown on figure 1.

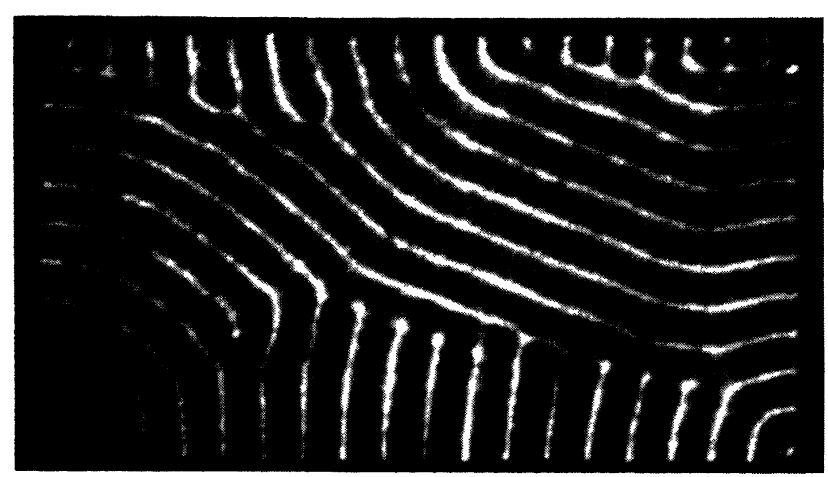

Fig. 1. - Focalization image of a Rayleigh-Bénard convective roll pattern obtained by slowly increasing the temperature gradient above its threshold. This so-called " natural structure " presents many defects and clearly illustrates the tendency of rolls to end perpendicularly to the sidewalls. $L x=38 d, L v=24 d, \varepsilon=0.88, P=70$. White lines correspond to descending flow and dark lines correspond to up-rising flow.

These structures undergo a slow time relaxation over a period of a few days [4] during wich the various defects present in the structure can move. Sometimes they disappear or nucleate or finally get trapped near a sidewall [3]. Dislocations are present in these texture-like structures, however they interact strongly with the other surrounding defects and, on the other hand, the wavenumber of the structure cannot be controlled. In such a situation a quantitative study of the dynamics of the dislocations is impossible.

Thus in order to perform the study we use " artificial structures $\gg$ obtained using the induction method described by M. M. Chen and J. A. Whitehead [27] : the desired pattern is obtained by triggering the convection by small thermal gradients induced in the oil in the slightly subcritical regime. These thermal gradients are obtained by illuminating the cell with a powerful light beam intercepted by a grid reproducing the desired structure. A sketch of the induction lay-out may be found in [22]. This method enables us to obtain regular patterns containing just one dislocation. However there is still one problem to overcome before the dislocation dynamics can be studied : namely an instability always occurs at the two shorter side-walls of the container. This instability, which has been previously investigated [3, 13], leads to the breakdown of the rolls adjacent to the sidewalls and replaces them by two sets of small rolls perpendicular to the sidewalls, as may be seen on figure 2. The occurrence of these two grain-boundary defects has a dramatic effect : they allow a continuous expansion or contraction of the structure leading to a very efficient wavevector selection as described in $\S 2.2$. This phenomenon would inhibit any study of the dependence of the dislocation dynamics on the wavenumber, which is an essential goal of this study.

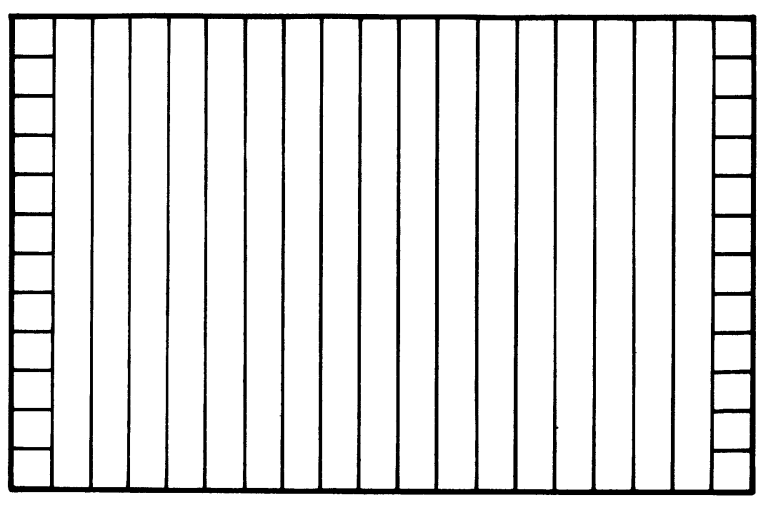

Fig. 2. - Sketch of the instability at the sidewalls leading to the occurrence of two grain-boundary defects in an induced structure. A picture of this phenomenon is given in reference 22 .

Fortunately we can get rid of this instability by altering the boundary conditions : we have glued two copper wires $0.5 \mathrm{~mm}$ in diameter along the two sidewalls. The copper wires, in thermal contact with the copper plate, trigger uprising flows at the shorter sidewalls. This device, already sucessful in the case of an axisymmetric pattern [3], stabilizes a structure with rolls parallel to the shorter sidewalls. Using this method, we were able to prepare, for a given Rayleigh number, stable structures with different wavevectors. In a way, this modification of the boundary conditions may give rise to a loose wavenumber selection [13, 14].

We now describe the exact pattern of the studied structures. Since we are using the two inductive wires, the structures have uprising flows at both ends. Thus they contain an even number of rolls, that is an integer number of wavelengths $N$. As the critical wavenumber corresponds to 19 wavelengths, we write the wavenumber of a structure as $k=N \times\left(k_{\mathrm{c}} / 19\right)\left(k_{\mathrm{c}}=3.117\right)$. In order to obtain an isolated dislocation we have prepared grids consisting in two sets of rolls whose wavenumbers differ by $k_{\mathrm{c}} / 19$, and we write these wavenumbers : $k_{1}=N \times\left(k_{\mathrm{c}} / 19\right)$ and $k_{2}=(N+1) \times\left(k_{\mathrm{c}} / 19\right)$. This configuration appears clearly on the grid presented on figure 3. Of course these two sets of rolls are in phase right at the sidewalls and in phase opposition at the middle of the container, where we want to produce the dislocation. Depending on the parity 


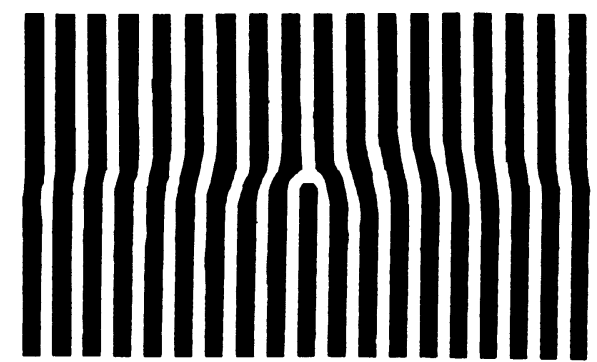

Fig. 3. - Induction grid for an isolated dislocation between two structures with the wavevectors $18 \times\left(k_{\mathrm{c}} / 19\right)$ and $19 \times\left(k_{\mathrm{c}} / 19\right)$.

of $N$, the flow at the core of the dislocation is either ascending or descending. Under Boussinesq conditions, we expect no modification of the dynamics. However it appears that the induction of a dislocation with an uprising flow at the core was easier to obtain. We suspect the grid drawing near the core to be responsible for this effect.

A typical experiment consists first in the induction of a dislocation pattern at a definite temperature, then a visual control of the overall pattern and finally an automatic dislocation tracking. This experimental process was not sucessful at each trial and we have encountered two kinds of problems, both related to the pattern. Some structural defects, undetectable during the visual control phase, could appear and produce a sudden change in the dislocation dynamics. But the major problem was the induction itself, due to two difficulties : first, the rising of the temperature produced an outgoing flow which tended to break the rolls close to the filling up hose and, second, our copper wires at the sidewalls were not perfectly efficient. This was probably due to unsatisfactory gluing of these wires. As a proof, the subcritical rolls expected were just visible. Consequently we could not stabilize structures with a wavevector too far from the optimal one.

Successful inductions were the result of several tries corresponding to various temperature rising rates and grid positions.

Comparatively, the determination of the dislocation velocity is rather simple but tedious. As a trial, we have followed the dislocation by taking a movie (one exposure every minute), but this proved to be a cumbersome method. Thus we have set up an automatic tracking technique to determine the dislocation position as a function of time.

For this purpose, we have used the focalization image of the structure. A parallel light beam entering the cell nearly vertically crossing the oil layer, and reflected by the highly polished copper plate, is finally sent on a screen. A photodiode placed on the screen enables us to analyse the image with an Apple II computer. The dislocation pattern, which appears on figure 4 , is recognized by moving the cell in front of the photodiode with two step by step motors. The experimental lay-out is depicted in figure 5 .

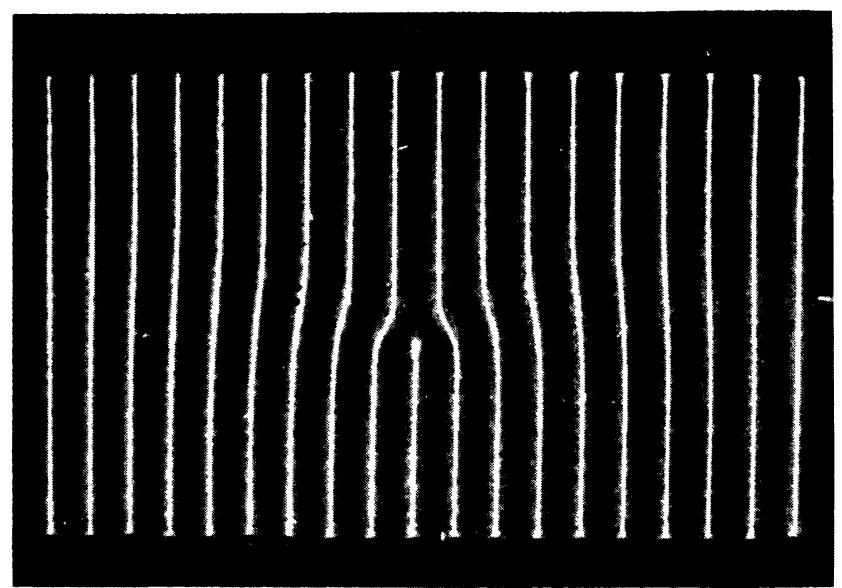

Fig. 4. - Focalization image of an isolated dislocation. $P=70, \varepsilon=0.5, K_{1}=18 \times\left(k_{\mathrm{c}} / 19\right)$ and $K_{2}=19 \times\left(k_{\mathrm{c}} / 19\right)$.

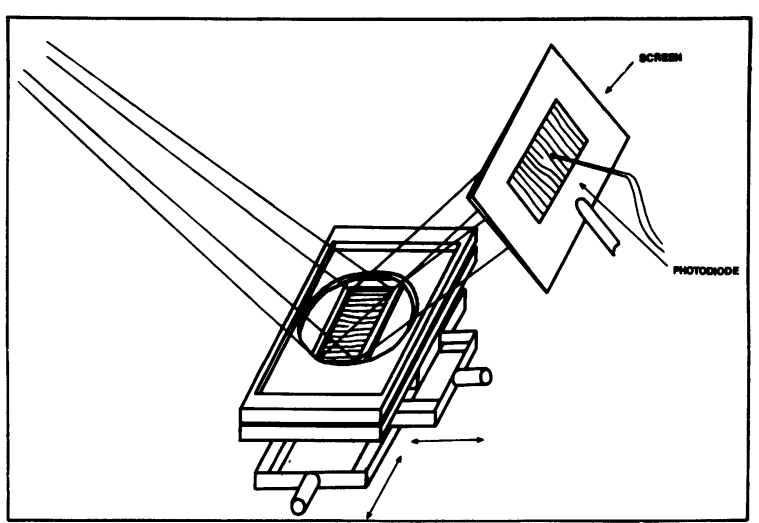

Fig. 5. - Sketch of the experimental lay-out : the experimental cell is placed on two step by step motors so that it may be moved at will under the control of an Apple II computer. The overall pattern is visualized using focalization image techniques, the intensity profile can be analysed using the photodiode placed at the centre of the screen.

As can be seen in figure 6, the dislocation pattern presents an intensity discontinuity at its core, along the roll direction and we have used it to determine the dislocation position. To identify this discontinuity, we scan the cell in front of the photodiode along the paths defined in figure 7. The paths A and B enable the computer to determine where to perform the last path $\mathrm{C}$ which reproduces the expected discontinuity. The detailed process used to determine the dislocation position is explained in the caption of figure 7. This scenario is iterated so that we get a data every thirty seconds. The accuracy on the dislocation displacement is about $0.05 \mathrm{~mm}$. The typical velocity of a defect being of the order of a few $\mathrm{cm} / \mathrm{hr}$., this technique proves to be perfectly adapted.

2.2 GRAIN BOUNDARY EXPERIMENTS. - In this experiment we have studied the wavenumber selection produced by a second kind of defect. As already mentioned, the rolls near the sidewalls have a tendency 


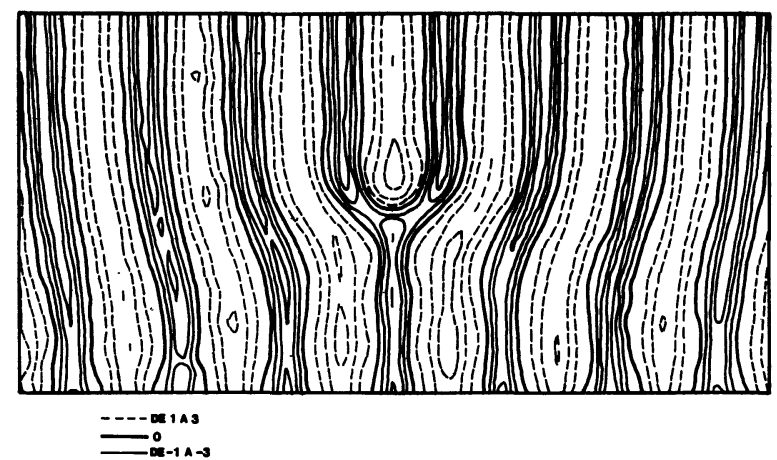

Fig. 6. - Intensity profile of the focalization image around a dislocation. Notice the intensity bump just at the dislocation core.

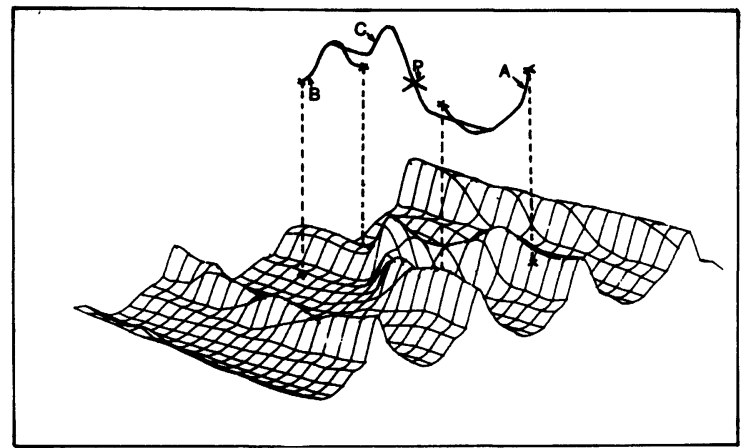

Fig. 7. - Scanning paths performed to determine the dislocation position. Path $\mathrm{A}$ : the computer searches the intensity minimum associated with the ascending flow. Path B : it looks for the maximum corresponding to descending flow. Then the computer scans on a line joining the minimum to the maximum (path $\mathrm{C}$ ). The abrupt intensity variation produced by the bump is recognized as the dislocation position (point $\mathrm{P}$ ). This process is repeated using the last position as the center of the new region of search.

to break up into rows of rolls perpendicular to the sidewalls [3], naturally producing two grain boundaries (Fig. 2). The length of these small rolls may change continuously giving to the main set of rolls the possibility of freely expanding or contracting. We have thus emphasized this property by inducing a pattern for which this expansion is not rapidly limited by the sidewalls, figure 8 , using the technique of Chen and Whitehead. In a way this is somewhat similar to Koschmieder's experiment [30], for in both experiments the selection occurs in a continuous manner. We may notice that since the structure presents rolls ending perpendicular to nearly all the sidewalls, the experiment was very simple to carry out. After the induction, we just increase the temperature step by step, and at each step, we measure the average wavelength, taking care of verifying that the wavelength evolution is finished. The average wavelength was determined automatically by scanning the index gradient field of the set of rolls parallel to the smaller side of the container.

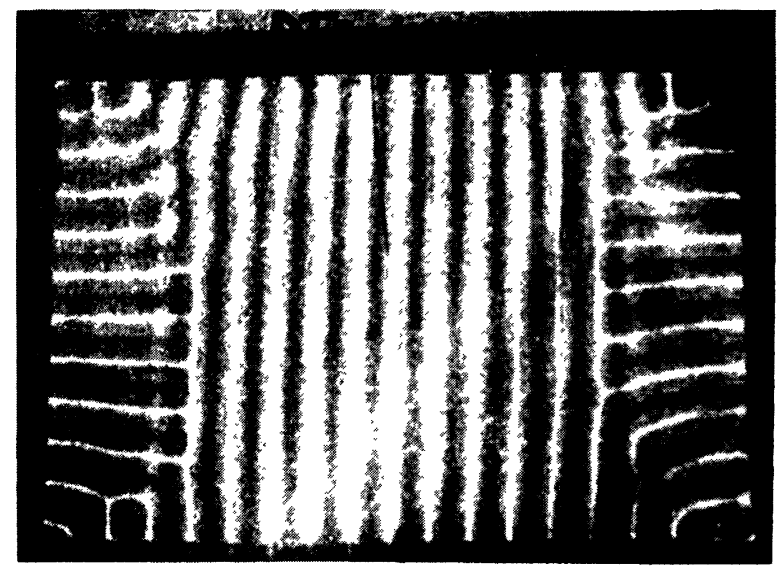

a)

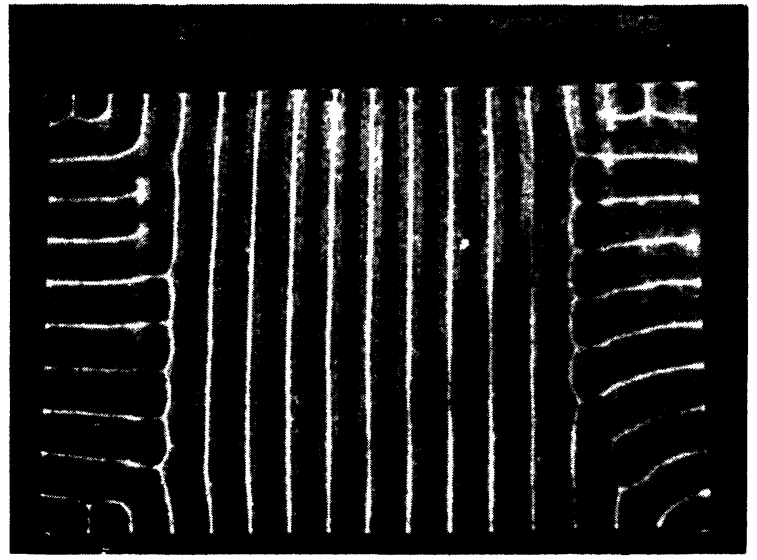

b)

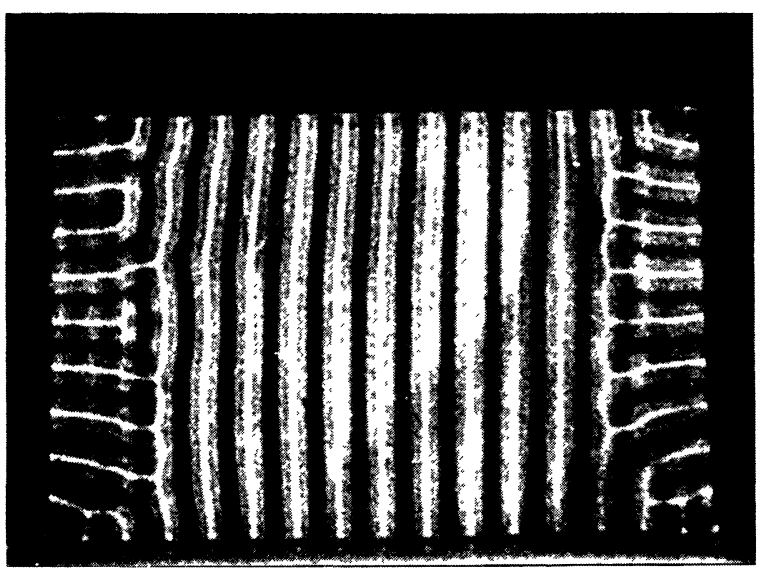

c)

Fig. 8. - Grain-boundary pattern used to determine the wavenumber selection curve. The two rows of lateral rolls enable the principal set of rolls to expand or to contract freely. Picture $a$ ) corresponds to $\varepsilon=1.30, b)$ to $\varepsilon=1.90$, c) to $\varepsilon=3.35$.

\section{Experimental results.}

\subsection{General CONSIDERations.}

3.1.1 Dislocation experiment. - From the symmetry of the pattern, one could expect climbing to be the only possible motion. In fact, this is the general rule in our experiments. However, we have also observed gliding motions, but they were limited to one roll distance and were probably due to either a misad- 
justement of the grid position with respect to the sidewalls or to the occurrence of structural defects. In fact the climbing motion constitutes a wavenumber selection mechanism : the dislocation moves so that, finally, only one wavevector remains in the cell. The selection follows a simple scheme : between the two wavevectors present simultaneously with the dislocation the pattern will choose the one closest to a well defined optimal wavevector. For instance, when $\varepsilon$ equals 1.5 , the optimal wavevector appears to be $17.5 \times\left(k_{\mathrm{c}} / 19\right)$. So, at $\varepsilon=1.5$, a dislocation between two structures having wavevectors $20 \times\left(k_{\mathrm{c}} / 19\right)$ and $21 \times\left(k_{\mathrm{c}} / 19\right)$ respectively will climb until only the structure with $K=20 \times\left(k_{\mathrm{c}} / 19\right)$ remains. Furthermore, the optimal wavevector decreases when the Rayleigh number increases, as can be, seen on figure 9 . Thus by changing

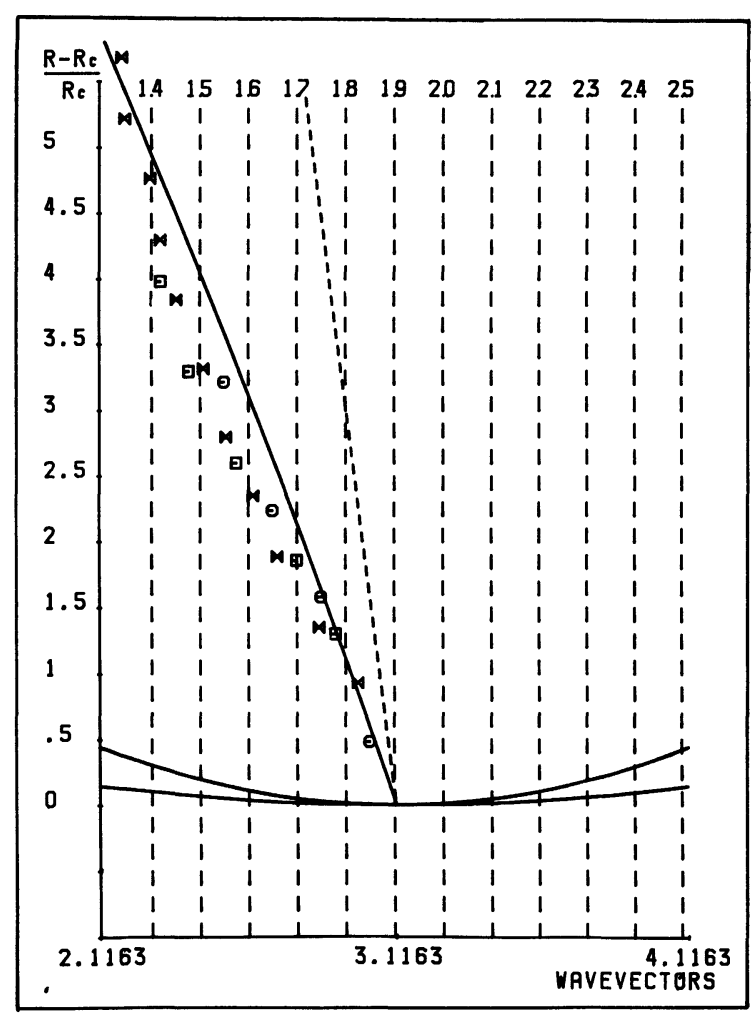

Fig. 9. - Experimental wavenumber selection compared to the $D_{\perp}=0$ criterion. The circles correspond to the zero dislocation velocity criterion. The wavenumber reported is an half-integer number which is the arithmetic value of the two wavenumbers of the structure. These points were determined by varying the Rayleigh number until the dislocation stops, or by extrapolation of results obtained at a fixed wavenumber. The squares are the velocity zeroes deduced at a constant Rayleigh number : from the fit of the various velocities obtained at the same Rayleigh number using the 1.5 power law for the wavevector variation we determine the wavenumber at which this velocity should cancel. The crosses correspond to the mean wave number of the central set of rolls of figure 8 , and thus give the wavenumber selection by grain-boundaries. The full-line is the computed $D_{\perp}=0$ curve at $P=70$ (marginal stability against zig-zag) and the dotted line is that for $D_{\perp}^{\prime}=0$ at the same Prandtl number (stationary axisymmetric convection). the Rayleigh number it is possible to reverse the velocity of a dislocation in the same structure and to move this defect back and forth, from one wall to the other. The condition is that the two wavevectors of the structure be the optimal ones for the appropriate Rayleigh numbers so that at a given Rayleigh number the structure prefers, say, the first wavevector, and at another Rayleigh number it prefers the second wavevector.

An interesting result, shown on figure $10 a$, is the uniformity of the dislocation's velocity during its travel towards the sidewall. This is true up to a short distance, of the order of one roll diameter, from the sidewall. This result is of great importance, since it enables quantitative measurements and comparisons with theoretical predictions $[18,19]$.

The effect of the sidewall is to decrease the velocity of the dislocation (see Fig. 10b), and when this velocity

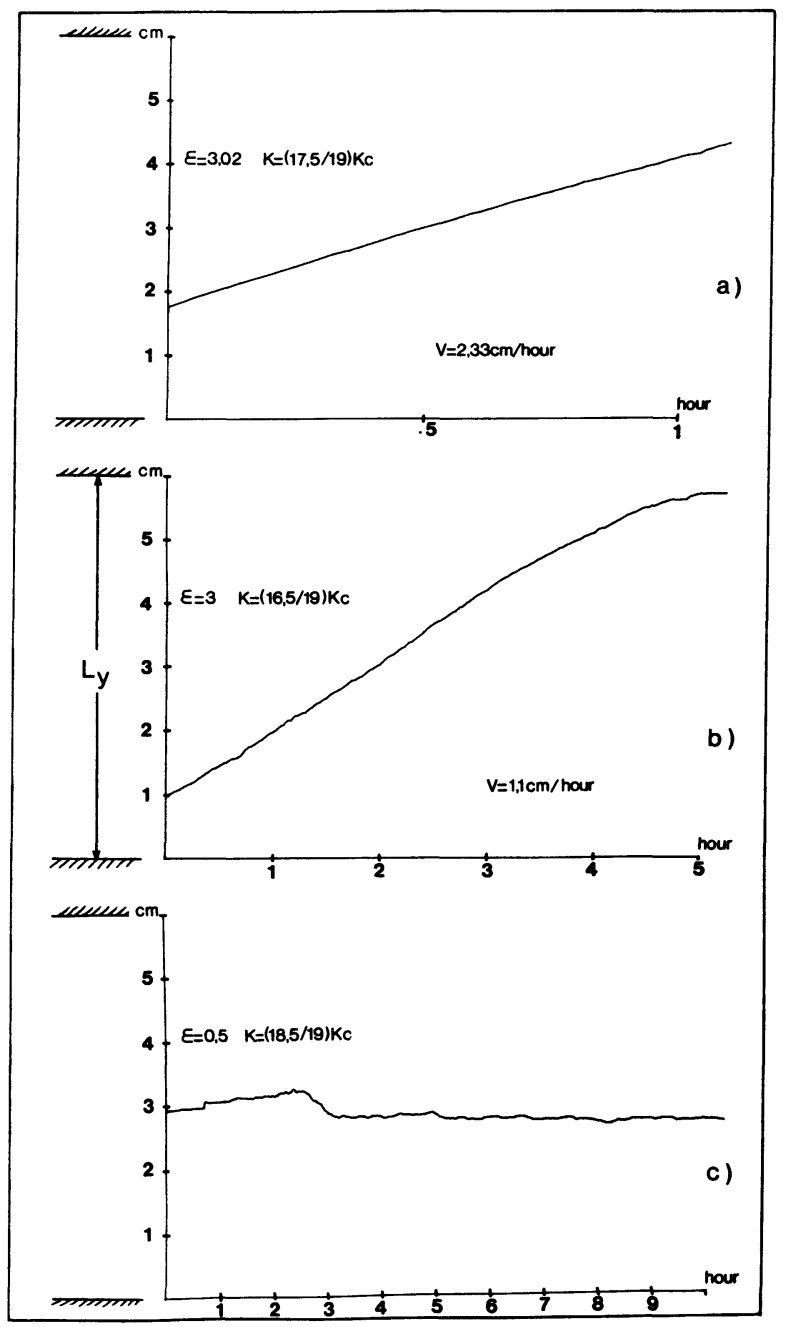

Fig. 10. - Recording of the dislocation position versus time. a) Uniform behaviour of the velocity, commonly observed; $P=70, k=17.5 \times\left(k_{\mathrm{c}} / 19\right), \varepsilon=3.02$. b) Influence of a sidewall : the dislocation is trapped at a short distance from the sidewall; $P=70, k=16.5 \times\left(k_{\mathrm{c}} / 19\right), \quad \varepsilon=3.00$. c) Very low velocity subjected to noisy variations; $P=70$, $k=18.5\left(k_{\mathrm{c}} / 19\right), \varepsilon=0.5$. 
is already small it can even immobilize it, so that the dislocation may be trapped at the sidewall (see Fig. 11).

The automatic tracking process gives us the position of the dislocation until it reaches the sidewall. We have considered that an experiment was successful if the velocity was constant during the complete climbing motion. With this criterion, we have eliminated experiments where a structural defect appears, which induces a noticeable change in the velocity. Nevertheless, we have noticed a strange behaviour when the velocity was very low (less than $0.5 \mathrm{~cm} / \mathrm{hr}$.). Though no defects other than the dislocation were visible, the velocity was not uniform and presented small abrupt variations as illustrated on figure $10 \mathrm{c}$. We cannot exclude some uncontrolled physical parameter being at the origin of this phenomena. Siggia and Zippelius's [18] have shown a similar phenomenon in their simulation, though in their case this could be due to the free slip boundary conditions used.

According to reference 19, the wavenumber selection curve should coincide with the curve $D_{\perp}=0$, in the $(k, \varepsilon)$ plane, at least for the variational case. This means that the zigzag instability appears for structures located on the left side of this curve figure 9. Once more, the experiment confirmed this point : most of the results that we present concern structures located on the right side where they were found to be stable,
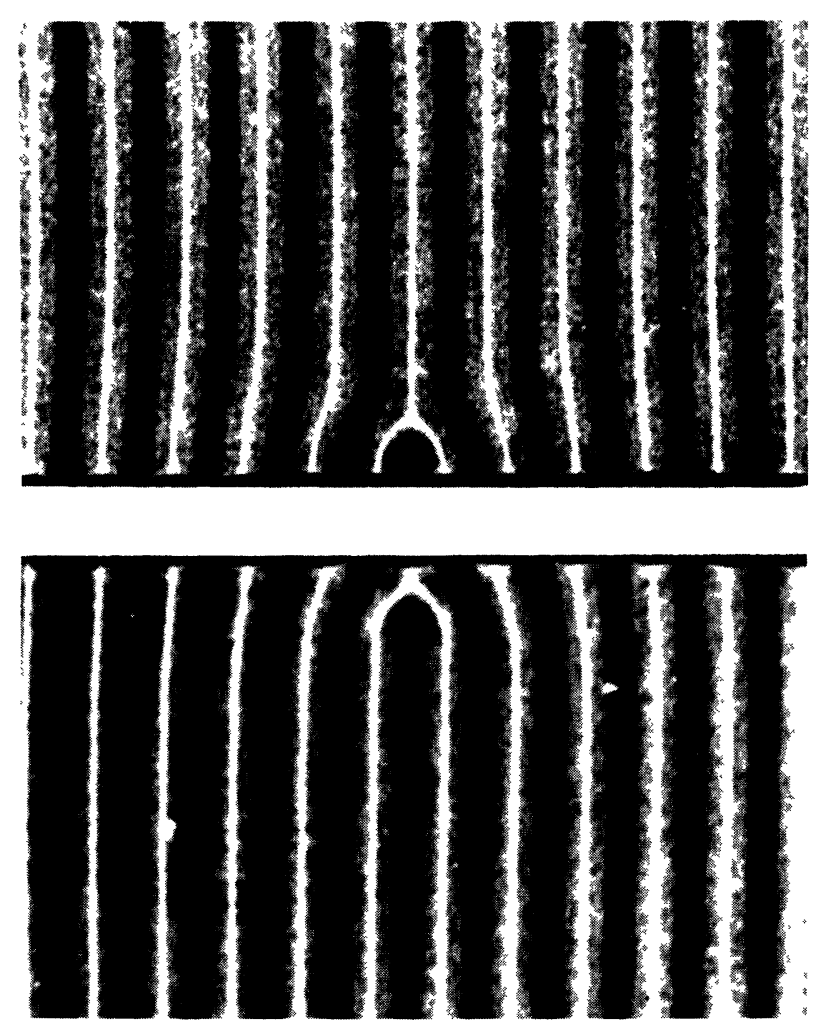

Fig. 11. - Trapped dislocation at the sidewall. When the dislocation velocity is small it may be stopped by the sidewall. The two pictures correspond to positive and negative velocity respectively. $P=70, k_{1}=17\left(k_{\mathrm{c}} / 19\right)$ and $k_{2}=18$ $\left(k_{\mathrm{c}} / 19\right)$. a) $\left.\varepsilon=1.8, b\right) \varepsilon=1.3$. while most of the others, on the left side, were unstable against zigzags. In fact, in this last case, the zigzag instability did not appear when the structure was close to the selection curve, so that negative dislocation velocities could be measured. The delay in the occurrence of the zigzag instability has already been observed [22] and is due to the finite size of the container.

The results that we present concern relatively high Rayleigh numbers, compared with the validity domain of theories $[18,19]$. We were surprised to notice that their main results seemed to hold at these Rayleigh numbers. This result was interesting by itself, but our motivation was also to obtain a noticeable wavenumber selection in order to compare with the wavenumber selection achieved in the grain boundary experiment.

3.1.2 Grain boundary experiment. - In this experiment we have found a continuous expansion of the wavelength with the Rayleigh number, until the central row of rolls completely filled the cell. The grain boundaries then disappeared and further wavenumber selection was inhibited.

The evolution of the wavenumber versus the Rayleigh number is shown by the cross shaped marks on figure 9. Clearly the selection is continuous and no noticeable hysteresis could be found by increasing and decreasing the Rayleigh number. During this experiment the zigzag instability did not occur.

\subsection{QUANTITATIVE RESUlts.}

3.2.1 Dislocation experiment. - Our experimental results are given in table I. Experiments with the same wavenumbers lie on vertical lines and those at the same reduced Rayleigh number lie on horizontal lines. For each experiment we have indicated the final wavenumber using an arrow and the value of the velocity in $\mathrm{cm} / \mathrm{hr}$. We estimate the accuracy on the reduced Rayleigh number to be about $1 \%$.

We must notice that the velocity increases as we go upwards or rightwards in table I. So that for wavevector smaller than $k_{\mathrm{c}}$ we can find a reduced Rayleigh number at which the velocity cancels. The wavenumber selection curve is then formed by the round and square shaped points on figure 9. The more distant the structure is from the wavenumber selection curve, the faster the dislocation moves. This result is consistent with the argument proposed in reference 19, i.e. the dislocation velocity is a function of $D_{\perp}$. The proof of this statement would be provided by the measurement of $D_{\perp}$ under the same experimental conditions. Though possible [22], this measurement is difficult to carry out and has not been performed here. Nevertheless we think that the statement is valid since the experimental wavenumber selection curve agrees fairly well with the line $D_{\perp}=0$ obtained from the calculations of Manneville and Piquemal [26]. This can be seen on figure 9. Once more we were surprised that this agreement remains very good even at relatively high Rayleigh 
Table I. - Dislocation velocity ( $\mathrm{cm} / \mathrm{hour}$ ).

\begin{tabular}{|c|c|c|c|c|c|c|c|c|}
\hline wav & & & & 8 & 20 & 21 & & $\begin{array}{l}\times\left(K_{c} / 19\right) \\
2\end{array}$ \\
\hline & & & & & & & & \\
\hline 4.76 & $\stackrel{2.6}{\longrightarrow}$ & & & & & & & \\
\hline 4.33 & $\frac{1.71}{1}$ & & & & & & & \\
\hline 4.05 & $\stackrel{1.72}{\perp}$ & & & & & & & \\
\hline 3.98 & 1.00 & 2.62 & 4.68 & 4.99 & 7.53 & & & \\
\hline 3.87 & $\stackrel{1.15}{\longrightarrow}$ & & & & & & & \\
\hline 3.63 & $\stackrel{0.74}{\circ}$ & & & & & & & \\
\hline 3.44 & 0.58 & & & & & & & \\
\hline 3.37 & & & 2.37 & $\stackrel{3.97}{\longrightarrow}$ & 5.15 & & & \\
\hline 3.29 & $\stackrel{0.19}{\longleftarrow}$ & $\frac{1.12}{\longleftarrow}$ & & & & & & \\
\hline 3.22 & $\stackrel{0}{\longrightarrow}$ & & & & & & & \\
\hline 3.15 & 0.11 & & & & & & & \\
\hline 3.09 & $\stackrel{0.10}{\longrightarrow}$ & & & & & & & \\
\hline 3.02 & & & $\stackrel{2.33}{\stackrel{2}{2}}$ & & & & & \\
\hline 2.67 . & & 0.36 & $\stackrel{1.82}{ }$ & 2.31 & 3.75 & 5.69 & 6.58 & \\
\hline 2.32 & & & $\stackrel{1.05}{\longrightarrow}$ & & & & & \\
\hline 2.22 & & & & & $\stackrel{3.13}{3}$ & & & \\
\hline 2.01 & & & 0.52 & & & & & \\
\hline 1.86 & & & $\stackrel{0.24}{\circ}$ & +.46 & 2.76 & 4.17 & & \\
\hline 1.70 & & & $\stackrel{0.12}{1}$ & & & & & \\
\hline 1.59 & & & $\stackrel{0.04}{\longrightarrow}$ & & & & & \\
\hline 1.55 & & & $\stackrel{0.09}{\longrightarrow}$ & & & & & \\
\hline 1.48 & & & $\stackrel{0.19}{\longrightarrow}$ & & & & & \\
\hline 1.44 & & & & & 1.76 & & & \\
\hline 1.37 & & & & $\stackrel{0.52}{\longrightarrow}$ & & & & \\
\hline 1.24 & & & & $\stackrel{0.40}{.4}$ & & 3.48 & & \\
\hline 0.62 & & & & $\stackrel{0: 05}{\longrightarrow}$ & $\stackrel{0.89}{\longrightarrow}$ & & & \\
\hline
\end{tabular}

numbers where perturbative theories are expected to break down.

This last point has motivated us to further compare our results with these theories. For this purpose, we have fitted them with an appropriate law for the velocity taken from reference 19 where it was found to be proportional to the $3 / 2$ power of $D_{\perp}$, which itself was found in reference 26 to be a linear function of the reduced Rayleigh number $\varepsilon$ and of the mean wavevector distance to the critical value $\mathrm{d} k$. Siggia and Zippelius [18] proposed an expression simply proportional to the $3 / 2$ power of $d k$. Our results show that the theoretical predictions of reference 19, which generalize Siggia's result by including the dependence on $\varepsilon$ are more appropriate.

Assuming :

$$
V=A[\mathrm{~d} k / B+C . \varepsilon]^{D}
$$

one can determine the experimental values of $A, B, C$ and $D$. The fit was performed by $\mathrm{M}$. Tournarie using a method described in [28] leading to the values quoted in table II.

We can appreciate the accuracy of the result, by considering the agreement with already known constants : $B$ should be the critical wavenumber 3.117 , and we find 3.186. We can also consider the slope of the line of zero velocity value $(\varepsilon=-4.90 \mathrm{~d} k)$. This value
Table II. - Numerical results of the fit

$$
V=A[\mathrm{~d} k / B+C \cdot \varepsilon]^{D} .
$$

$A=36.45(\mathrm{~cm} / \mathrm{h})$

Root mean square

$B=3.186$ 4.3

$C=0.064$

$3 \times 10^{-3}$

$D=1.23$

$1 \times 10^{-3}$

0.16

is in good agreement with the theoretical slope $(\varepsilon=-6.94 \mathrm{~d} k)$ determined in [26].

The interesting result of this fit is the power law exponent $D$ since both theories $[18,19]$ predict it to be 1.5 at least in the variational and linear phase dynamic approximation. In fact, we found that the best exponent for our experimental results is 1.23 with a root mean square deviation of 0.16 . Nevertheless we cannot exclude the value 1.5 , since the determination of the parameters $A$ and $D$ proved to be strongly correlated : while the accuracy in $B$ and $C$ is good, that of $A$ and $D$ is poor. Then the meaning of the root mean square 0.16 is somewhat misleading; it indicates the best choice distribution probability but does not take into account the correlation between $A$ and $D$. To illustrate this well known behaviour we have imposed the value of the exponent $D=1.5$ in the fit, the corresponding parameters are reproduced in table III. The quality factors of the two fits proved to be almost the same, meaning that the 1.5 value is almost as good as the best value 1.23 and that the determination of the exact exponent is not possible with our experimental results. However an accurate determination of the exponent would be interesting since it would provide a quantitative test of perturbative methods.

Table III. - Numerical results of the fit

$$
V=A[\mathrm{~d} k / B+C . \varepsilon]^{D}
$$

with $D$ imposed to 1.5 .

$A=49.06(\mathrm{~cm} / \mathrm{h})$

Root mean square

$B=3.13$

$C=0.066$

$3 \times 10^{-3}$

$1 \times 10^{-3}$

$D=$ is imposed at the value 1.5 .

Such a determination has been made by Siggia and Zippelius in reference 18, however for this purpose they have used simulations of the amplitude equation which just verify the analytical calculation based on the same equation. No check had been performed in the Boussinesq simulations, which could have reflected a contribution of the vertical vorticity for example. This aspect is of importance since the vertical vorticity is related to secondary flows [5] in the perturbative analysis and to the appearance of non-variational terms in the amplitude equation [24]. 
Using the value obtained for $A$ in our fit it is possible to compute the equivalent of the beta factor introduced by Siggia and Zippelius in [18] :

$$
A=\beta \frac{\xi_{\|}^{2}}{\tau} \frac{k_{\mathrm{c}}}{\sqrt{2}}
$$

Which leads in physical units to :

$$
\beta=A \frac{d}{\kappa} \frac{1+0.5117 P^{-1}}{6.4098}
$$

For the fit of table II, we get $\beta=0.58$, and for table III $\beta=0.78$. This numerical factor has been determined by Siggia and Zippelius in [18]. Our results are in relatively good agreement with the $\beta=0.84$ value that they proposed.

The Rayleigh-Bénard problem is indeed nonvariational, but for some experimental conditions (high Prandtl and low Rayleigh numbers) the behaviour of the convection does not clearly reflect this point. On the other hand, in the low Prandtl number case, the perturbative methods are not satisfactory [24]. We can then ask whether there is a crossover between the two situations. In our point of view, we think that the non-variational character will appear in a limited manner for a moderate Prandtl number. If we consider the theoretical analysis of reference 19 the difference which appears in the nonvariational model compared to the variational model consists in the separation between the wavenumber selection curve corresponding to zero dislocation velocity value and the curve $D_{\perp}=0$.

This effect does not appear in figure 9 due to its poor resolution. However, to construct the curve $v=0$, we have performed a detailed study of this region. Knowing approximately, for a defined pair of wavenumbers, the Rayleigh number at which the dislocation stays at rest, we have induced this structure at this Rayleigh number, and then measured its velocity as a function of the Rayleigh number which was varied in a small range. Although the values of the velocities are small and thus slightly noisy, they exhibit a strange behaviour as may be seen on figure $12 a$. We guess that the rapid variation of the velocity with the Rayleigh number is due to the effect of the curve separation quoted above. Its extension is small in terms of Rayleigh number, and as expected gets smaller at lower Rayleigh numbers (see Fig. 12b).

3.2.1 Grain boundary experiment. - This experiment is straightforward; the selected wavenumber is given

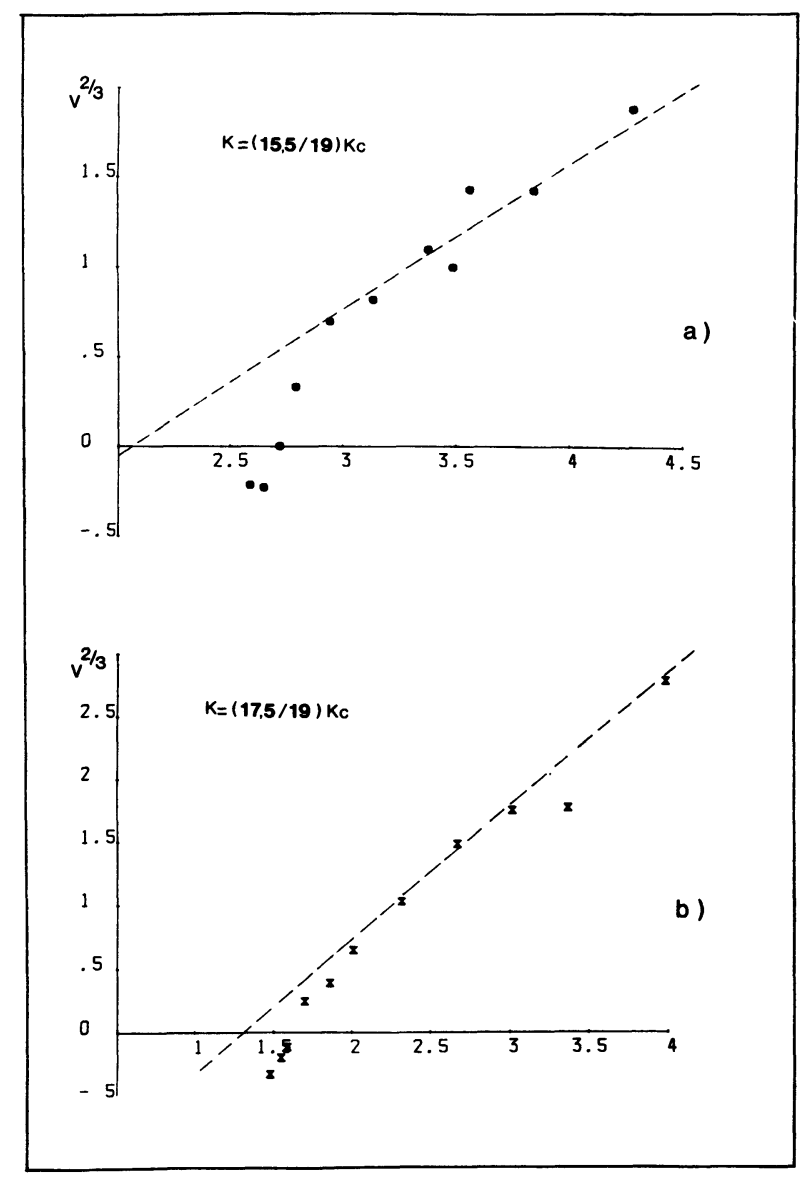

Fig. 12. - Dislocation velocity to the power $2 / 3$ versus the reduced Rayleigh number at a fixed wavevector. In such graphs, points should be aligned if the system behaved according to equation 2 . In fact this is true only when the velocity is large enough, and the experimental points then tend asymptotically to a linear behaviour, roughly proportional to $D_{\perp}$. When the velocity is small this is no longer the case so that the zero of velocity differs from the $D_{\perp}=0$ criterion. a) The wavevector is fixed at $15.5\left(k_{\mathrm{c}} / 19\right)$. The discrepancy between the zero extrapolated from the asymptotical line and the zero of velocity may be evaluated at 0.65 on the reduced Rayleigh number. b) The wavevector is fixed at $17.5\left(k_{\mathrm{c}} / 19\right)$. The effect is less pronounced than in a) but, as expected, the separation between the extrapolated zero and the velocity zero, indicates a convergence of the selection curves at the threshold.

in table IV as a function of the Rayleigh number and is reproduced as crosses on figure 9 which definitely shows that this selection criterion cannot be distinguished from the others. However the grain boundary

Table IV. - Wavenumber selection obtained with the grain-boundary defects.

\begin{tabular}{|c|r|r|r|r|r|r|r|r|r|r|c|}
\hline$\frac{R-R_{\mathrm{c}}}{R_{\mathrm{c}}}$ & 0.93 & 1.35 & 1.89 & 2.35 & 2.80 & 3.32 & 3.84 & 4.30 & 4.77 & 5.22 & 5.68 \\
\hline wavevector $\times\left(K_{\mathrm{c}} / 19\right)$ & 18.26 & 17.46 & 16.60 & 16.10 & 15.54 & 15.08 & 14.52 & 14.19 & 13.98 & 13.45 & 13.39 \\
\hline
\end{tabular}


motion provides a faster selection mechanism than the dislocation motion. Thus, when a grain boundary appeared in a dislocation structure, it released the stress quickly so that the dislocation stopped moving.

\section{3 Discussion OF THE Results of J. A. White-} HEAD. - As already pointed out, Whitehead's experiment was somewhat different from ours : he studied the velocity of a row of dislocations joining two roll structures with wavelengths in the ratio $3 / 2$. In such an experiment, the dislocations are very close to each other so that their strain fields interact strongly, as may be seen in pictures 1 of reference 23. Nevertheless, this experiment gives us valuable information, i.e. the dependence of the dislocation velocity on the Prandtl number. Using three different fluids at the same Rayleigh number $(\varepsilon=9.6)$ and the same mean wavevector $\left(k \simeq k_{\mathrm{c}}\right.$ ) but with different Prandtl numbers, Whitehead found that the normalized dislocation velocity $V d / \kappa$ roughly scales like $P^{-1}$.

According to our experiments we suggest that this result is tightly connected with the wavenumber selection mechanism : for relatively large Prandtl numbers $(P>10)$, which is indeed the case of Whitehead's experiments, the wavenumber selection and thus the locus of dislocation velocity zero should coincide with the line $D_{\perp}=0$ to a first approximation. This line crosses the marginal stability curve in the $(\varepsilon, k)$ plane at the point $\left(0, k_{\mathrm{c}}\right)$ whatever the Prandtl number; its slope is negative and increases like $P$, so that it is nearly vertical when $P=\infty$. As the velocity of the dislocation varies as the power $3 / 2$ of the distance between its representing point in the plane $(\varepsilon, k)$ and the line $D_{\perp}=0$, this leads to the following expression for the dislocation velocity within the assumptions $\varepsilon \ll 1, P \rightarrow \infty$ :

$$
\begin{aligned}
\frac{V d}{\kappa}=\beta & \cdot \frac{6.4098}{1+0.5117 P^{-1}} \times \\
& \times\left(\frac{0.16591+23.095 P^{-1}}{10.76}\right)^{3 / 2} \cdot(\varepsilon)^{3 / 2} .
\end{aligned}
$$

Using this expression 4 we find velocities which are smaller than those reported by J. A. Whitehead and a Prandtl number dependence which varies from $P^{-1}$ to $P^{-3 / 2}$. But Whitehead's experiment is far away from the validity domain of (4).

Another interpretation of Whitehead's findings concerning the Prandtl number dependence is proposed by E. D. Siggia [29] : the large scale flows which generate vertical vorticity [24] should account for the defect velocity; as these flows depend strongly on $P$ one should expect that the dislocation velocity scales like $\varepsilon^{7 / 4} / P$.

At present we cannot determine which interpretation is correct, more accurate experiments at different Prandtl numbers would be welcome.

3.4 DISCUSSION CONCERNING THE WAVENUMBER SELECTION. - If we consider the wavenumber selection curves on figure 9, we may be surprised at the very good agreement between the experimental results and the theoretical curve $D_{\perp}=0$. Although it seems natural to obtain stationary dislocations when $D_{\perp}=0$, the numerical formulation used for $D_{\perp}$ remains valid only when the secondary flows [24] are not counteracted by pressure gradients. Cross [30] and Manneville and Piquemal [26] have pointed out that these secondary flows which were not included in the previous amplitude equations [8-10] modify the phase diffusion equation or at least its coefficients. However in a finite container, these secondary flows are associated with pressure gradients in order to insure the fluid conservation $[30,26]$. Considering the zigzag instability, owing to the divergence-free character of the secondary flows, Cross indicates that these pressure gradients should not be taken into account. In this case P. Manneville and J. M. Piquemal have calculated the expression of the diffusion coefficient $D_{\perp}$ [26]. In our opinion, the question remains open whether or not these secondary flows are partially counteracted by the sidewalls supressing the divergence free condition, an extreme case being the axisymmetric situation where the counteracting effect is complete. In this last case, Manneville and Piquemal have found a selection criterion which is different from $D_{\perp}=0$ and which we can denote $D_{\perp}^{\prime}=0$ by analogy.

Owing to that situation, we could have inferred that the effective coefficient acting in the dislocation case might be a compromise between $D_{\perp}$ and $D_{\perp}^{\prime}$. However, it seems that the selection occurs effectively at $D_{\perp}=0$, in agreement with Cross's predictions for the zigzag instability boundary. If the secondary flows seems not to be counteracted in the dislocation and grain boundaries case, they certainly are counteracted in the axisymmetric situation. Thus we can propose an experimental check of the existence of the secondary flows which consists in the measurement of the wavenumber selection in an axisymmetric structure using the same Prandtl number as for the dislocation experiment $(P=70)$. In this case the selection mechanism described by Y. Pomeau and P. Manneville [15] should be efficient and the selected states should lie on the curve $D_{\perp}^{\prime}=0$. At this Prandtl number value $(P=70)$, this curve is sufficiently different from the selected curve obtained here $\left(D_{\perp}=0\right)$ to be experimentally distinguished (see Fig. 9).

Such an experiment has already been performed by E. L. Koschmieder [31], but the Prandtl number of the fluid was too large $(P=511, P=916)$ to allow a clear cut difference to be seen between the $D_{\perp}$ and $D_{\perp}^{\prime}=0$ curves. Furthermore, the sidewalls in this experiment probably induced a strong up-rising flow which stabilized the axisymmetric pattern, and also altered the wavenumber selection. This is likely since the wavenumber selected at the critical Rayleigh number was found to be different from the critical wavenumber.

It is worth noticing that if we consider the experi- 
ment of Willis, Deardoff and Sommerville [32] in the large Prandtl number case $(P=450)$ the slope of the wavenumber selection that they proposed is also in reasonable agreement with the curve $D_{\perp}=0$ calculated for this Prandtl number.

In contrast with the results of this last paper [32], dislocations or grain boundary defects allow to well defined, hysteresis-free, wavenumber selection to be obtained. Such accurate methods are necessary in order to distinguish between the various wavenumber mechanisms which have been proposed [13-17, 33, 34].

In order to test the validity of the agreement between the wavenumber selection by dislocations or grain boundaries with the curve $D_{\perp}=0$, we tried to reproduce the experiments at a smaller Prandtl number $(P=13)$, all other experimental conditions being kept identical. In this case the structure appeared to be extremely unstable. The stabilizing temperature gradients at the side-walls were inefficient so that we could not keep a uniform structure without defects near the sidewalls. Further attempts using the same oil proved that, with more powerful temperature gradients at the sidewalls, the experiment could be successfully carried out. This is in progress in our laboratory presently.

\section{Conclusion.}

In this paper, we have demonstrated that it is possible to prepare an isolated dislocation in a large RayleighBénard structure and that a pattern can be made compatible with the sidewalls of the container in order to facilitate its study. The evolution of this structure consists in the motion of the dislocation along the rolls' axis, releasing the stress present in the structure. Although the evolution of the pattern is driven by this motion, the dislocation dynamics are tightly connected to the overall structure. We have found that dislocation climb occurred with a uniform velocity, which was a fundamental hypothesis of theoretical works $[18,19]$. This velocity increases with the wavenumber as predicted in references 18 and 19 and with the Rayleigh number as in reference 19. Although our experiments dealt with Rayleigh numbers which are outside the validity domain of the present theories $[18,19]$ the velocity variation could be written as a power law, though the experimental exponent $(1.23 \pm 0.16)$ differed slightly from the theoretical value (1.5).

A very important concept, already suggested by F. H. Busse and J. A. Whitehead [34], is that dislocation motion offers a wavenumber selection mechanism. In a way, a dislocation gives the structure the possibility to choose between two wavenumbers, and its motion favours the wavenumber which is closer to some " optimal » value. At this " optimal " wavenumber the dislocation velocity vanishes. The curve $v=0$ defines the wavenumber selection line in the $(k, \varepsilon)$ plane. We found that for a value of $\varepsilon$ smaller than five this curve is a straight line, starting at the critical wavenumber at the convection threshold, and leaning towards smaller wavenumbers. This experiment provides an accurate and unambiguous determination of this curve, and allows for a quantitative comparison with theoretical predictions $[18,19]$. Our experimental curve coincides with the criterion $D_{\perp}=0$ introduced in [16]. To test the universality of this criterion, we have designed a second experiment in which the wavenumber selection mechanism occurs via grain boundary defects. This mechanism appears to be very efficient and is as accurate as the dislocation mechanism. The corresponding selection curve confirmed the $D_{\perp}=0$ criterion.

Contrary to what occurs in ordinary crystals, Rayleigh-Bénard structures are not governed by any variational principle. At the relatively high Prandtl number at which this experiment was achieved $(P=70)$ the nonvariational character does not seem to be dominant but it may be at the origin of the peculiar behaviour of the dislocation velocity near the selection curve.

\section{Acknowledgments.}

We wish to acknowledge P. Manneville, Y. Pomeau, P. Bergé, M. Dubois, J. M. Piquemal, S. Zaleski, P. Tabeling, J. E. Wesfreid, E. Guazelli, E. Guyon, E. Dubois-Violette for stimulating discussions. We have also greatly benefited from the series of seminars organized by S. Fauve at l'Ecole Normale Supérieure. We are indebted to C. Poitou, M. Labouise and B. Ozenda for their valuable technical assistance. We wish also to acknowledge M. Tournarie who performed the numerical fits.

\section{References}

[1] Dubois, M., Bergé, P., J. Physique 42 (1981) 643.

Bergé, P., Dubois, M., Manneville, P. Pomeau, Y., J. Physique Lett. 41 (1980) L-341.

Dubois, M., Bergé, P. and Croquette, V., J. Physique Lett. 43 (1982) L-295.

Maurer, J. and LibChaber, A., J. Physique Lett. 41 (1980) L-515.

Libchaber, A. and Maurer, J., J. Physique Colloq. 41 (1980) C3-51.
Libchaber, A., Laroche, C. and Fauve, S., J. Physique Lett. 43 (1982) L-211.

Giglio, M., Muzzati, S. and Perini, U., Phys. Rev. Lett. 47 (1981) 243.

Gollub, J. P. and Benson, S. V., J. Fluid. Mech. 100 (1980) 499.

[2] Ruelle, D. and Takens, F., Comm. Math. Phys. 20 (1971) 167.

Feigendaum, M. J., J. Stat. Phys. 19 (1978) 25. 
Manneville, P. and Pomeau, Y., Physica D 1 (1980) 219.

[3] Croquette, V., Mory, M. and Schosseler, F., J. Physique 44 (1983) 293.

[4] Gollub, J. P., McCarriar, A. R. and Steinman, J. F.,

J. Fluid Mech. 125 (1982) 259.

Gollub, J. A. and Steinman, J. F., Phys. Rev. Lett. 47 (1981) 505.

Gollub, J. P. and McCarriar, A. R., Phys. Rev. A 26 (1982) 3470.

[5] BERGÉ, P. in Chaos and Order in Nature H. Haken ed. (Springer Verlag, Berlin) 1981 p. 14.

[6] Ahlers, G. and Behringer, R. P., Phys. Rev. Lett. 40 (1978) 712.

Ahlers, G. and Walden, R. W., Phys. Rev. Lett. 44 (1980) 445.

[7] Libchaber, A. and Maurer, J., J. Physique Lett. 39 (1978) L-369.

[8] Segel, L. A., J. Fluid Mech. 38 (1969) 203.

Cross, M. C., Phys. Fluids 23 (1980) 1727.

[9] Wesfreid, J., Pomeau, Y., Dubois, M., Normand, C., Bergé, P., J. Physique 39 (1978) 75.

[10] Newell, A. C. and WhiteheAd, J. A., J. Fluid Mech. 38 (1969) 279.

[11] Cross, M. C., Phys. Rev. A. 25 (1982) 1065.

[12] Greenside, H. S., Coughram, Jr., W. H., Schryer, N. L., Phys. Rev. Lett. 49 (1982) 726.

[13] Pomeau, Y. and Zaleski, S., J. Physique 42 (1981) 515.

[14] Cross, M. C., Daniels, P. G., Hohenberg, P. C. and Siggia, E. D., Phys. Rev. Lett. 45 (1980) 898 and J. Fluid Mech. 127 (1983) 155.

Cross, M. C., Hohenberg, P. C., Safran, S. A., Physica D 5 (1982) 75.

[15] Pomeau, Y. and Manneville, P., J. Physique 42 (1981) 1067.

[16] Pomeau, Y. and Manneville, P., J. Physique Lett. 40 (1979) L-609.
Pomeau, Y. and Manneville, P., Phys. Lett. A 75 (1980) 296.

[17] Kramer, L., Ben-Jacob, E., Brand, H. and Cross, H. C., Phys. Rev. Lett. 49 (1982) 1891.

[18] Siggia, E. D. and Zippelius, A., Phys. Rev. A. 24 (1981) 1036.

[19] Pomeau, Y., Zaleski, S. and Manneville, P., Phys. Rev. A 27 (1983) 2710.

[20] Dubois-Violette, E., Guazzeli, E., Prost, J., Philos. Mag. $A$ (to appear).

[21] Manneville, P. and Pomeau, Y., Philos. Mag. $A$ (to appear).

[22] Croquette, V. and Schossler, F., J. Physique 43 (1982) 1183.

[23] Whitehead, J. A., J. Fluid Mech. 75 (1976) 715.

[24] ZipPelius, A. and Siggia, E. D., preprint NSF ITP 82 42.

Siggia, E. D. and Zippelius, A., Phys. Rev. Lett. 47 (1981) 835.

[25] Friedel, J., Les dislocations (Gauthier-Villars ed., Paris) 1956.

[26] Manneville, P. and Piquemal, J. M., Phys. Rev. A 28 (1983) 1774.

[27] Chen, M. M. and Whitehead, J. A., J. Fluid Mech. 31 (1968) 1 .

[28] Tournarie, M., J. Physique 30 (1969) 737.

[29] Siggia, E. D., private communication.

[30] Cross, M. C., Phys. Rev. A 27 (1983) 490.

[31] Koschmieder, E. L. and Pallas, S. C., J. Heat. Mass. Trans. 17 (1974) 991.

[32] Willis, G. E., DeardorfF, G. W. and Sommerville, R.C.J., J. Fluid Mech. 54 (1972) 351.

[33] Clever, R. M. and Busse, F. H., J. Appl. Math. Phys. 29 (1978) 711.

[34] Busse, F. H. and Whitehead, J. A., J. Fluid Mech. 47 (1971) 305. 